\title{
Consumo de sustancias psicoactivas y su relación con comportamiento violento en estudiantes universitarios
}

\section{Consumption of psychoactive substances and its relationship with violent behavior in university students}

\author{
CAMPO, Yarlis M. ${ }^{1}$ \\ OLIVEROS, Jhon J. ${ }^{2}$ \\ AMED, Eustorgio J. ${ }^{3}$
}

\begin{abstract}
Resumen
La progresiva participación de jóvenes en consumo de sustacias psicoactivas (SPA) y algunas formas de violencia se presentan como un problema en incremento; esta investigación tuvo como objetivo determinar la relación entre consumo de sustancias psicoactivas y comportamientos violentos en estudiantes universitarios de Sincelejo (Colombia). Se desarrolló un estudio transversal con fase correlacional, tomando una muestra aleatoria de 492 estudiantes aplicando un análisis descriptivo e inferencial de las variables estudiadas. Se concluye que el consumo de SPA se relaciona débilmente con comportamientos violentos. La heroína, éxtasis y el consumo simultáneo de ácidos y marihuana representan mayor influencia en los eventos violentos.

Palabras clave: violencia, consumidores de drogas, salud del estudiante, correlación de datos.
\end{abstract}

\begin{abstract}
The progressive participation of young people in the consumption of psychoactive substances (SPA) and some forms of violence presents an increasing problem; This research aimed to determine the relationship between consumption of psychoactive substances and violent behaviors in university students from Sincelejo (Colombia). A cross-sectional study with a correlational phase was developed, taking a random sample of 492 students applying a descriptive and inferential analysis of the variables studied. It is concluded that the consumption of SPA is weakly related to violent behaviors. Heroin, ecstasy, and the simultaneous consumption of acids and marijuana represent the greatest influence in violent events.
\end{abstract}

Key words: violence, drugs users, student health, correlation of data.

\section{Introducción}

La creciente participación de la población juvenil en el consumo de sustancias psicoactivas y actos delictivos o de alguna forma de violencia, ha sido uno de los problemas más acuciantes en los últimos tiempos (Oficina de Naciones Unidas contra la Droga y el Delito [UNODC], 2018). Este fenómeno social binomial se ha asumido con carácter de axioma, mostrándose desde una compleja naturaleza, pero con un gran impacto en las personas y

\footnotetext{
${ }^{1}$ Fonoaudióloga. Magister en Salud Pública. Universidad de Sucre. Email: yarliscampop21@gmail.com

${ }^{2}$ Médico. Especialista en Seguridad Social. Magister en Salud Pública. Universidad de Sucre. Email: jhox17@hotmail.com

${ }^{3}$ Enfermero. Magister en Salud Pública. Docente Investigador. Universidad de Sucre. Facultad Ciencias de la Salud. Email: eustorgio.amed@unisucre.edu.co
} 
comunidades, alterando sublimemente, el desarrollo de las mismas (Ortega et al., f 2018). En el panorama mundial, el consumo de drogas resulta una situación complicada por el fenómeno de policonsumo, dada la tendencia a utilizar más de una sustancia psicoactiva (SPA) simultánea o sucesivamente (UNODC, 2016). Según la Organización Mundial de la Salud (OMS) de 155 a 250 millones de personas (3.5\% a 5.7\% de la población mundial) entre los 15 y los 64 años habrían usado algún tipo de sustancia psicoactiva, como cannabis, anfetamina, cocaína, opioide o medicamentos no prescritos. Actualmente, se estima que alrededor de 15.3 millones de personas en el mundo sufren de algún trastorno psicosocial o físico por uso de sustancias(Observatorio de Drogas de Colombia [ODC], 2016).

Según el informe mundial sobre las drogas, para el 2014, el número de muertes relacionadas con drogas fue de 43,5 por millón de personas de entre 15 y 64 años; datos que involucran actos delictivos y de violencia social (UNODC, 2016).

Se reporta además el aumento de consumo de sustancias de origen desconocido que se suministran por cauces ilícitos y se hacen pasar por medicamentos, cuando en realidad están destinadas al consumo con fines no médicos, lo cual sin dudas, se convierte en una situación alarmante para la salud física y mental de los consumidores, mencionando también las consecuencias que su consumo acarrea, entre ellos, su involucramiento en la ocurrencia de conductas violentas de alguna manera(ODC, 2013; UNODC, 2018).

A nivel continental, el consumo de drogas entre los adolescentes de América es muy agudo y la percepción de riesgo frente al uso ocasional de esas sustancias es muy baja. La investigación elaborada por la Comisión Interamericana para el Control del Abuso de Drogas (CICAD) de la Organización de Estados Americanos (OEA), revela el alto consumo de alcohol y marihuana en jóvenes de entre 13 y 17 años y la precocidad en el consumo de éxtasis y sustancias inhalables(Comisión Interamericana para el Control del Abuso de Drogas [CICAD]., Secretaría de Seguridad Multidimensional., 2015).

Desde el enfoque de salud pública, es claro que todas las drogas presentan riesgos para la salud de las personas que las consumen. Bajo esta perspectiva, en Colombia, el gobierno nacional promueve la formulación del "Plan Nacional de Promoción de la Salud, Prevención y Atención del Consumo de Drogas 2014 - 2021", que se fundamenta en los enfoques de la salud pública y de derechos humanos y que fue aprobado por el Consejo Nacional de Estupefacientes(Observatorio de Drogas de Colombia, 2016). Estudios recientes han demostrado que los adolescentes que consumen alcohol antes de los 14 años pueden tener hasta 10 veces más riesgo de presentar problemas sociales y de salud con el alcohol en la adultez, así como de otras sustancias(Pérez, A., Scoppetta, 2008).

Se agrega que, durante este mismo año, el promedio nacional de prevalencia del consumo de alcohol o de drogas ilícitas fue de 3,6 por 100.000 habitantes y mientras que la prevalencia para el departamento de Sucre durante la misma vigencia fue de 1,4 por 100.000 habitantes, siendo una cifra preocupante, si se compara con la tasa de base nacional(ODC, 2013).

Por su parte, en el municipio de Sincelejo, pese a que la problemática resulta latente, existen escasos registros estadísticos del consumo de drogas. Un estudio efectuado en estudiantes de la Universidad de Sucre en el 2016, reporta que el $87,4 \%$ ha consumido alcohol, tabaco y un $70,9 \%$ drogas ilegales bajo fines sociorecreativos (en celebraciones, eventos sociales); mientras que el cigarrillo manifiestan fumarlo una vez por semana(Amed et al., (2016). Con relación al consumo y producción de SPA, informes de la policía afirman que los barrios del norte de Sincelejo que más sufren por este delito son Cielo azul, Camilo Torres, Altos del Rosario y Cristo viene; y en el sur, La Trinidad, Villa Mady y El Poblado (ODC, 2013). Del mismo modo, según los Indicadores de Seguridad 2017 - 2018, del ministerio de defensa, la tasa de homicidios en los últimos 12 meses es de 13,4, lesiones personales 
con un $32 \%$ y con un $34 \%$ el hurto a personas, residencias y comercios; siendo todas estas modalidades actos disruptivos de criminalidad y formas de violencia(Ministerio de Defensa, 2017).

Dada la problemática presentada, se hace necesario abordar en los nexos del consumo de sustancias SPA y los actos violentos, entendiendo como primera medida que en su presentación participan múltiples factores que obligan a que las alternativas de solución sean articuladas desde diferentes sectores y con una mirada integradora. Así, desde el presente estudio, se abordó en la población juvenil universitaria, dada la complejidad de rápido cambio biológico y socio-cultural que convierte a este sector de la comunidad vulnerable a estos fenómenos sociales, que generan un impacto eminente y agravante para la salud de la sociedad en general.

\section{Metodología}

\subsection{Tipo de estudio}

Se llevó a cabo un estudio descriptivo, con diseño transversal y una fase correlacional (Díaz, 2009), orientado a establecer la relación entre el consumo de sustancias psicoactivas (SPA) y los comportamientos violentos en los estudiantes de educación superior del municipio de Sincelejo, durante el presente año.

\subsection{Universo, población y muestra}

El universo del presente estudio estuvo constituido por todos los estudiantes de educación superior del departamento de Sucre; la población fue conformada por 13.767 estudiantes de educación superior en pregrado, adscritos a seis 6 instituciones de educación superior con sede en el municipio de Sincelejo. El método a utilizar fue aleatorio simple. La selección de los estudiantes se hizo a través de muestreo aleatorio simple, donde se tuvieron en cuenta todas las facultades y programas de pregrados de las instituciones de educación superior anteriormente seleccionadas. Se calculó el tamaño de la muestra para poblaciones finitas, obteniendo un tamaño muestral de 374 individuos, con un nivel de confianza del $95 \%$ y margen de error del $5 \%$.

Los criterios para selección de la muestra fueron: Encontrarse inscritos y activos como estudiantes en algún programa de pregrado en las instituciones de educación superior seleccionadas, acceder a través del consentimiento informado a la realización de la encuesta. Los criterios de exclusión fueron: No encontrarse activos en calidad de estudiantes en las instituciones seleccionadas, autodeclarado de dificultades visuales, auditivas o motoras que interfieran en la realización de la encuesta y presencia de condiciones premórbidas asociadas.

\subsection{Fuente de información}

Fuente primaria con aplicación de cuestionario de Google Forms en línea, enviado a los correos electrónicos y números telefónicos vía WhatsApp de cada estudiante, a través de un enlace URL para su fácil difusión y respuesta. Se determinó una tasa de respuesta del instrumento del $37.6 \%$.

\subsection{Instrumento}

Se aplicó un formulario estructurado como técnica de recolección, con preguntas de tipo dicotómicas, politómicas y de jerarquización (Escala de Likert). Algunas preguntas fueron extraídas o tomadas de estudios anteriores similares(Chavarriaga, M., Segura, 2015; Instituto Nacional de Psiquiatría Ramón de la Fuente Muñiz [INPRFM], 2015; UNODC, 2017), basadas en factores sociodemográficos que se asocian al consumo de sustancias psicoactivas, así como referidas a comportamientos violentos. Se realizaron las de pruebas de validez y fiabilidad del instrumento de medición, a través de un panel de 3 expertos. Posteriormente, se llevó a cabo la aplicación de la prueba piloto a 50 estudiantes. El cuestionario final se ensambló con los ítems que presentaron alta confiabilidad estadística, dando como resultado un Alfa de Cron Bach de 0,97. 
Su aplicación final se llevó a cabo en el periodo comprendido entre el mes de febrero y mayo del presente año.

\subsection{Análisis estadístico}

Se llevó a cabo un análisis descriptivo de las características sociodemográficas, académicas, y de condiciones de vida; así mismo, se determinó la prevalencia de consumo de SPA de los jóvenes y se describieron los comportamientos violentos de los estudiantes en calidad de víctimas o victimarios. Para el análisis de estadístico inferencial, se dispuso del software y programa estadístico $R$, versión 3.3.2. Se aplicó la técnica de Chi-cuadrado para relacionar las variables de consumo de SPA y comportamientos violentos. Para medir la intensidad de la relación entre las variables de estudio, se usó el Coeficiente de Cramer, calculado en Microsoft Excel 2016. Se estimaron los intervalos de confianza (IC 95\%) del haber sido autor de comportamientos violentos con el consumo de SPA.

Por último, se realizó un análisis multivariado, aplicando la técnica de análisis de correspondencias múltiples (ACM) para abordar todas las variables categóricas del fenómeno de consumo de SPA y su relación con los comportamientos violentos presentados.

\section{Resultados}

Para el desarrollo de este estudio, se contó con la participación total de 723 estudiantes de educación superior. Atendiendo a los criterios de selección de la muestra y objetivos de la investigación, se tomó una muestra de 492 estudiantes que manifiestan consumir sustancias psicoactivas, correspondientes al $68.04 \%$.

\subsection{Prevalencia del consumo de sustancias psicoactivas en los estudiantes universitarios}

\section{Gráfico 1}

Prevalencia de vida y prevalencia anual según consumo de sustancias psicoactivas en Universitarios, Sincelejo. 2020

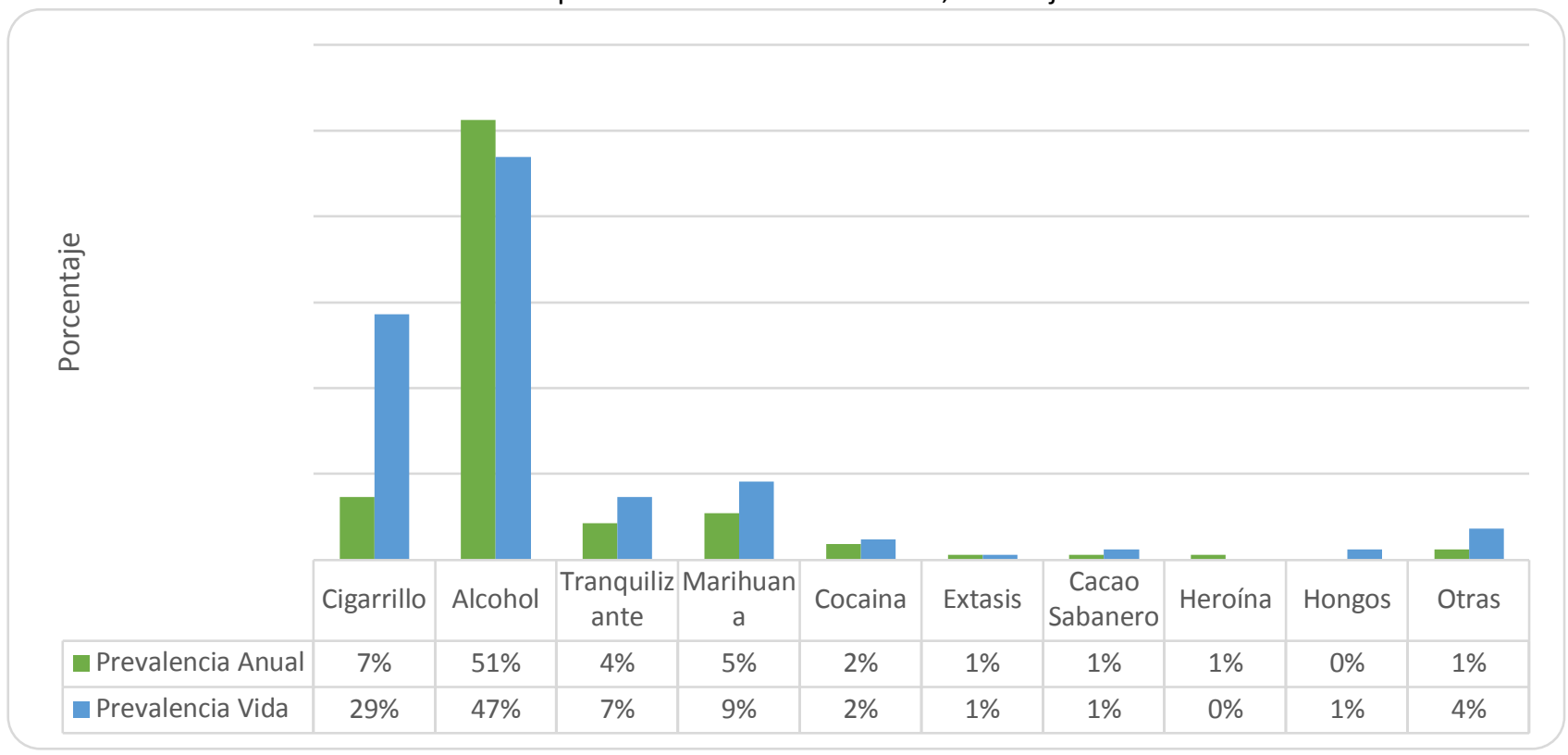

Fuente: Elaboración propia de los autores

Atendiendo a la prevalencia de consumo anual de sustancias psicoactivas de los estudiantes universitarios (Fig. 1), se encontraron las bebidas alcohólicas, como las mayormente consumidas durante el último año (51\%), seguido del cigarrillo (7\%); siendo éstas las sustancias de uso legal. En tercera posición, se encuentra la marihuana (5\%) y el uso de tranquilizantes (4\%). 
En cuanto al consumo de vida, se reportaron bajo la misma jerarquía, las bebidas alcohólicas (47\%), cigarrillo (29\%), marihuana (9\%) y tranquilizantes (7\%).

Tabla1

Variables sociodemográficas

\begin{tabular}{|c|c|c|}
\hline Variable & Frecuencia & Porcentaje \% \\
\hline \multicolumn{3}{|l|}{ SEXO } \\
\hline Femenino & 285 & 58 \\
\hline Masculino & 207 & 42 \\
\hline \multicolumn{3}{|l|}{ EDAD } \\
\hline $15-18$ años & 57 & 11.6 \\
\hline $19-22$ años & 285 & 58 \\
\hline 23-26 años & 111 & 22.6 \\
\hline 27-30 años & 21 & 4.3 \\
\hline Mayor de 31 años & 18 & 3.6 \\
\hline \multicolumn{3}{|l|}{ ESTRATO } \\
\hline 1 & 285 & 58 \\
\hline 2 & 144 & 29.2 \\
\hline 3 & 48 & 9.7 \\
\hline 4 & 9 & 1.81 \\
\hline 5 & 6 & 1.21 \\
\hline \multicolumn{3}{|l|}{ LUGAR } \\
\hline Sincelejo & 318 & 64.63 \\
\hline Otro municipio de Sucre & 147 & 29.87 \\
\hline Fuera del dpto. Sucre & 21 & 4.26 \\
\hline $\begin{array}{l}\text { Zona Periférica de } \\
\text { Sincelejo }\end{array}$ & 6 & 1.21 \\
\hline \multicolumn{3}{|l|}{ ESTADO CIVIL } \\
\hline Soltero (a) & 453 & 92.07 \\
\hline Casado (a) & 15 & 3.04 \\
\hline Separado (a) & 12 & 2.43 \\
\hline Unión Libre & 12 & 2.43 \\
\hline \multicolumn{3}{|l|}{ ÁREAS DE ESTUDIO } \\
\hline Ciencias de la Salud & 108 & 21.95 \\
\hline $\begin{array}{l}\text { Ciencias Económicas y } \\
\text { Administrativas }\end{array}$ & 171 & 34.75 \\
\hline Educación y Ciencias & 192 & 39.02 \\
\hline Ingeniería & 21 & 4.26 \\
\hline \multicolumn{3}{|l|}{ SEMESTRE } \\
\hline 1 & 60 & 12.19 \\
\hline 2 & 18 & 3.65 \\
\hline 3 & 42 & 8.53 \\
\hline 4 & 15 & 3.04 \\
\hline 5 & 27 & 5.48 \\
\hline 6 & 36 & 7.31 \\
\hline 7 & 87 & 17.68 \\
\hline 8 & 54 & 10.97 \\
\hline 9 & 69 & 14.02 \\
\hline 10 & 72 & 14.63 \\
\hline 11 & 6 & 1.21 \\
\hline 12 & 6 & 1.21 \\
\hline
\end{tabular}

Fuente: Elaboración propia de los autores

Con base al consumo de SPA por género, se obtuvo que la población femenina manifiesta consumir mayormente (58\%), respecto a la masculina (42\%). En cuanto a las edades, se halló que los estudiantes de 19 a 22 años se encuentran en primer lugar, frente al consumo de SPA (58\%), seguido de los educandos de 23 a 26 años (22.6\%) y la categoría de 15 a 18 años (11.6\%). En menor proporción, el rango de 27 a 30 años (4.3\%) y mayores de 31 
años (3.6\%). De esta manera, se exhibe una participación considerable en el consumo de SPA, en edades más tempranas en los estudiantes universitarios (Tabla 1 ).

Con respecto al nivel socioeconómico, se muestra que, la mayor parte de la población estudiantil se concentró en los estratos más bajos: estrato 1 (58\%), seguido del estrato 2 (29.2\%); y estrato 3 (9.7\%). De acuerdo al lugar de residencia, se encontró que la mayoría de los estudiantes universitarios que manifiestan consumir SPA, viven en el municipio de Sincelejo (64.63\%) y están solteros (92.07\%). El área de estudio de mayor concentración fue el de educación y ciencias (39\%).

La participación de los estudiantes correspondió a todos los semestres, notando una mayor agrupación en los semestres superiores, del VII al X con el 57.3\%, \%, a excepción del XII y XII con un acumulado del 2.42\%. Para los primeros semestres, desde el I hasta el VI se presentó con un $40.2 \%$ (tabla 1 ).

Tabla 2

Consumo de SPA según el sexo de los estudiantes de educación superior del municipio de Sincelejo. 2020

\begin{tabular}{|l|c|c|c|c|}
\hline \multicolumn{1}{|c|}{ Sustancia SPA } & Frecuencia & Masculino N(\%) & Femenino N(\%) & Razón (H/M) \\
\hline Bebidas alcohólicas & 483 & $204(42.2)$ & $279(57.8)$ & 0.73 \\
\hline Cigarrillo & 177 & $93(52.5)$ & $84(47.4)$ & 1.1 \\
\hline Tranquilizantes & 57 & $33(57.8)$ & $24(42.10)$ & 1.3 \\
\hline Marihuana & 72 & $36(50)$ & $36(50)$ & 1 \\
\hline Cocaína & 21 & $15(71.4)$ & $6(28.6)$ & 2.5 \\
\hline Éxtasis & 6 & $6(100)$ & $0(0)$ & 0 \\
\hline Cacao Sab. & 9 & $6(66.7)$ & $3(33.3)$ & 2 \\
\hline Heroína & 3 & $3(100)$ & $0(0)$ & 0 \\
\hline Hongos & 6 & $0(0)$ & $6(100)$ & 0 \\
\hline Otras & 24 & $9(37.5)$ & $15(62.5)$ & 0.6 \\
\hline
\end{tabular}

Fuente: Elaboración propia de los autores

En concordancia con el consumo por cada sustancia SPA según el sexo de los estudiantes universitarios, se muestra que, por cada 2.5 hombres que consumen cocaína existe 1 mujer que lo hace; por cada 2 hombres que usan cacao sabanero, 1 mujer concurre consumiéndolo; siendo considerable estos datos.

Se observa también que, en el consumo de tranquilizantes, cada 2.6 hombres consumidoras de esta sustancia, hay 2 mujeres efectuando dicho consumo. Para el caso del cigarrillo y la cocaína, se muestra una distribución normal, en la que, por cada hombre consumidor de estas SPA, existe 1 mujer consumidora para cada una de ellas. Para las bebidas alcohólicas, la situación es contraria, pues es mayor la proporción de mujeres que consumen licor, cervezas, vinos, entre otros, respecto a los hombres, en la que por cada 2.19 hombres que consuman esta SPA, existen 3 mujeres ingiriéndolas (tabla 2).

Atendiendo a la frecuencia con la que los estudiantes de educación superior consumen SPA, se denota un comportamiento bastante irregular en la periodicidad del consumo de estas sustancias; sin embargo, se refleja una mayor concentración de consumo entre cada mes a cada seis meses (68.89\%). Seguido a esto, el consumo más esporádico, entre el anual y cada 2 años (23.16\%). Los estudiantes que reportaron un consumo más recurrente, entre diario a cada 15 días, se encontraron con menor afluencia (7.89\%).

De la población estudiada, el 75\% refiere no consumir de manera simultánea SPA; sin embargo, el 25\% reportan hacerlo con bebidas alcohólicas y marihuana, alcohol y cigarrillo, marihuana y Tusi, alcohol y Popper, marihuana y LS2, entre otras.

Se encontró además, que el $\mathbf{8 8 . 4 \%}$ de los estudiantes manifiesta voluntad y dominio propio para abstenerse a dicho consumo. No obstante, el $11.5 \%$ restante, revela poca facilidad para hacerlo, vislumbrando cierta 
drogodependencia. Al contrastar estas apreciaciones con la fecha de último consumo, se muestra que, el $62.15 \%$ ha presentado el uso de SPA desde el tiempo más reciente hasta los 3 meses, mientras que el 37.72\% reporta un consumo menos reciente y con ello frecuente, según este criterio.

El 78\% de estudiantes universitarios no reportan haber presentado problemas en sus relaciones interpersonales generado por consumo de SPA. En cuanto a la ayuda familiar/social y médica/profesional que los educandos manifiestan haber solicitado frente al consumo de SPA, se encontró que, para ambos tipos de apoyo, la mayoría no accede a su asistencia. Así, el $\mathbf{9 8 . 1 7 \%}$ no acude a ayuda familiar o social y el $96.34 \%$ no solicita apoyo en servicio médico o profesional.

Atendiendo a los síntomas físicos de abstinencia del consumo de SPA, se encontró que gran parte de la población estudiantil universitaria, refiere no presentarlos (95.73\%). Sólo un 4.24\% manifiesta presentar síntomas como sudoración, taquicardia, ansiedad, entre otros, cuando ha dejado de consumir alguna sustancia SPA.

Al estudiar el consumo de SPA por orden médica, se encontró que la mayoría de los estudiantes lo hace con fines socio recreativos (97\%). Sólo una minoría afirmó consumirlas por precepto médico (3\%). Entre las sustancias SPA mencionadas por los educandos están: marihuana, tranquilizantes, fluoxetina, codeína y antidepresivos

\subsection{Identificación de comportamientos violentos en los estudiantes universitarios y su grado de severidad}

Dentro de los comportamientos de violencia destacados bajo efectos del consumo de SPA (Tabla 3), se observó que el insulto o humillación de manera directa fue el evento violento principalmente reportado (14.01\%), seguido de golpes con un objeto (9.74\%). Llama la atención, el suicidio en tercera posición (6.08\%) y la acción de disparar con arma de fuego (6\%). La amenaza verbal y las acciones de autoagresión o daño físico se presentan con un $5.48 \%$. De este modo, se detallan formas de violencia de naturaleza verbal, física y en función de la víctima, interpersonal y autoinflingida.

Con relación a los comportamientos de violencia efectuados por los estudiantes universitarios sin estar bajo efectos de SPA, se encontró en primer lugar el insulto de manera directa (32.3\%), golpes con objetos (26.78\%) y amenaza verbal (16.48\%), siendo éstas formas de violencia de naturaleza verbal y física. Resulta importante resaltar las acciones de autoagresión (12.17\%) y el suicidio (11\%), como formas de violencia autoinflingida. En menor proporción se presentan formas de violencia cibernética, como los insultos por redes sociales (9.73\%).

Atendiendo a la presentación de eventos violentos en los que, los estudiantes fueron víctima, se encuentra que el $55.48 \%$ no ha sido víctima. No obstante, el $44.49 \%$ sí lo ha sido. Esta situación particular, muestra una realidad un poco indistinta.

\subsection{Relación entre el consumo de SPA y la participación en actos violentos}

Tabla 3

Resultados de Prueba $\chi^{2}$ (Chi- cuadrado) para determinar relación del consumo de SPA y comportamientos violentos

\begin{tabular}{|c|c|c|c|c|c|c|c|}
\hline & & \multicolumn{2}{|c|}{ Comportamiento violento } & & \multirow[t]{2}{*}{ IC95\% } & $\mathrm{x}^{2}(\mathrm{P}-$ value $)$ & Coeficiente \\
\hline \multirow{4}{*}{$\begin{array}{l}\text { Consumo } \\
\text { de SPA }\end{array}$} & & Violentos & No violentos & Total & & \multirow{4}{*}{$28.27(1.055 e-7)$} & \multirow{4}{*}{0,1977} \\
\hline & Consumidores & 220 & 272 & 492 & (5.8\%, 9.8\%) & & \\
\hline & $\begin{array}{c}\text { No } \\
\text { consumidores }\end{array}$ & 55 & 176 & 231 & $(27.1 \%, 33.9 \%)$ & & \\
\hline & Total & 275 & 448 & 723 & & & \\
\hline
\end{tabular}

Fuente: Elaboración propia de los autores 
Al medir la relación de consumo de SPA y comportamientos violentos, se tomaron los valores de la población consumidora y con respuesta positiva a comportamientos violentos y la población no consumidora que presentó igualmente conductas de violencia. Al emplear la prueba $\chi^{2}$, se encuentró un valor de $p=1.055 e-7$, evidenciando significancia estadística, considerando $p \leq 0,05$. De esta manera se rechaza la hipótesis nula y se acierta la relación existente entre el hecho de ser consumidor de SPA y presentar como efecto, comportamientos violentos (tabla 3).

Se establecieron los intervalos de confianza del 95\% (IC95\%) para cada grupo, encontrando que si se estudia la totalidad de la población estudiantil de educación superior, del $5.8 \%$ al $9.8 \%$ de los educandos no consumidores, presentarían actos violentos. Por su parte, del $27.1 \%$ al 33.9\% de en calidad de consumidores de SPA, ostentarían acciones de violencia; notando bastante considerable estos valores de proporción poblacional.

Se calculó la intensidad de la relación entre las variables de estudio, hallando un Cramer's V=0.1977; que expresa una relación débil entre el consumo de SPA y los comportamientos violentos en los estudiantes universitarios de Sincelejo. Esto podría indicar que, aunque la relación entre las variables de estudio es acierta, la presentación de los comportamientos violentos en los estudiantes universitarios del municipio de Sincelejo, podría estar relacionado a otros factores y no fundamentalmente al consumo de SPA.

Tabla 4

Resultados de Análisis de Correspondencias Múltiples por dimensiones estudiadas

\begin{tabular}{|l|l|r|}
\hline Bajo SPA & \multicolumn{1}{|l|}{ R2 } & P-value \\
\hline Ha insultado, difamado por redes sociales o publicaciones web & 0.89998087 & $3,98 \mathrm{e}-241$ \\
\hline Ha amenazado a alguien para obligarlo a cambiar de casa & 0.89998087 & $3,98 \mathrm{e}-241$ \\
\hline Ha amenazado a alguien con cuchillo o navaja & 0.89998087 & $3,98 \mathrm{e}-241$ \\
\hline Ha herido a alguien con cuchillo o navaja & 0.89998087 & $3,98 \mathrm{e}-241$ \\
\hline Ha amenazado a alguien con arma de fuego & 0.89998087 & $3,98 \mathrm{e}-241$ \\
\hline Sin SPA & $\mathbf{R 2}$ & P-value \\
\hline Ha amenazado a alguien para obtener sustancias psicoactivas & 0.89516288 & $3,28 \mathrm{e}-234$ \\
\hline Ha amenazado a alguien para obligarlo a cambiar de casa & 0.89516288 & $3,28 \mathrm{e}-234$ \\
\hline Ha amenazado a alguien con arma de fuego & 0.89516288 & $3,28 \mathrm{e}-234$ \\
\hline Ha intentado violar a alguien & 0.89516288 & $3,28 \mathrm{e}-234$ \\
\hline Ha violado a alguien & 0.89516288 & $4,14 \mathrm{e}-136$ \\
\hline Ha retenido a alguien & 0.73591233 & $4,14 \mathrm{e}-136$ \\
\hline Por variable y respuesta & Estimate & P-Value \\
\hline Estrato_3 & & 2,29 \\
\hline Edad de 23 a 26 años & $3,44 \mathrm{e}-54$ \\
\hline Consumo: Cada uno a cinco meses & 1,06 & $1,57 \mathrm{e}-18$ \\
\hline
\end{tabular}

Fuente: Programa estadístico R versión 3.3.2

Los comportamientos de violencia más relacionados con los estudiantes universitarios que consumen SPA y se encuentran bajo efectos de la misma son: insultar o difamar por redes sociales o publicaciones web, amenazar a alguien para obligarlo a cambiar de casa, amenazar con el uso de armar corto punzantes como cuchillo o navaja, herir a una persona con cuchillo y disparar con arma de fuego (Tabla 4).

Por su parte, se muestra que, sin estar bajo efectos de SPA, los comportamientos que presentan relación y significancia estadística en los estudiantes universitarios del municipio de Sincelejo son: amenazar a alguien para obtener SPA, amenazar a una persona para obligarlo a cambiar de residencia, amenazar a alguien con arma de fuego, intentar violar a una persona, cometer abuso sexual y retener a un individuo. Cabe resaltar que, aunque estas conductas de violencia tienen relación frente al hecho de ser consumidores, su significancia no es igual o mayor a las presentadas estando bajo efectos de las SPA. 
Las variables que mayor valor estimado y significancia estadística presentan son los estudiantes estrato 3 , edades de 23 a 26 años y la frecuencia de consumo de 1 a 5 meses.

\section{Caracterización de los grupos de acuerdo a los niveles de respuestas relacionadas a partir del Análisis de Correspondencias Múltiples}

Desde la caracterización ofrecida por los resultados, se evidencia que desde la dimensión 1 aparecen 3 grupos tales como los siguientes:

El grupo 1 (Consumidores violentos) caracterizado por una coordenada positiva en el eje, está formado por factores de alta frecuencia: comportamiento violento a persona con sustancia bajo el efecto de sustancia psicoactiva, último consumo hace menos de una semana, consumo simultáneo de SPA. Sin estar bajo efectos de estas sustancias, este grupo de estudiantes han robado algunas veces, y han humillado de forma directa. Se encuentra la presencia del estrato 3 y el semestre 9.

El grupo 2 (Consumidores no violentos) caracterizado por una coordenada negativa en el eje, está formado por factores de alta frecuencia: no presentan comportamientos de violencia, e incluso su violencia es nula bajo efectos de SPA, edades entre 27 y 30 años, con un último consumo de 2 a 3 meses, ubicados en semestres 5 y 8 y estrato socioeconómico 1. No presentan problemas de tipo social y legal frente al consumo de SPA.

El grupo 3 (Consumidores abstinentes no violentos) está formado por individuos que comparten factores de alta frecuencia: No presentan comportamientos violentos bajo efectos de SPA, estrato socioeconómico 2 y semestre 2, con menos de 15 días de haber consumido de drogas, con facilidad de dejar de consumirlas, no presenta policonsumo simultáneo.

Tabla 5

Resultados de Wilks Test

\begin{tabular}{|rrrrr|}
\hline$\# \#$ & Frecuencia_consumo & Semestre Cuales_Mismo_Tiempo & humilla_sinsus \\
$\# \#$ & $3.718359 \mathrm{e}-30$ & $1.887449 \mathrm{e}-23$ & $4.268785 \mathrm{e}-21$ & $7.631582 \mathrm{e}-19$ \\
$\# \#$ & sus_ord_med & Ultima_Vez & Problemas_consumo & robado_sinsus \\
$\# \#$ & $3.143883 \mathrm{e}-15$ & $6.678626 \mathrm{e}-15$ & $2.483063 \mathrm{e}-13$ & $6.721522 \mathrm{e}-13$ \\
$\# \#$ & suicidio_sinsus & Estrato & Arrestado_Drogas & Consumo \\
$\# \#$ & $1.394400 \mathrm{e}-11$ & $1.411378 \mathrm{e}-10$ & $5.782467 \mathrm{e}-10$ & $5.073379 \mathrm{e}-\theta 9$
\end{tabular}

Fuente: Programa estadístico R versión 3.3.2

Los resultados del Wilks test (Tabla 5), permiten indicar qué factores separan mejor el plano. En este caso la frecuencia de consumo quien es la que muestra una mayor significancia estadística $(p \leq 0,05)$. Para efectos del fenómeno de violencia, el factor que más distancia las personas violentas de las que no lo son, es la frecuencia de consumo de SPA en los estudiantes universitarios. 


\subsection{Principales SPA que influyen en el comportamiento violento de los estudiantes de educación superior}

Tabla 6

Sustancias psicoactivas que influyen según la caracterización por los 3 grupos

\begin{tabular}{|c|c|c|c|}
\hline \multirow{2}{*}{ Variable } & \multicolumn{3}{|c|}{ Correlación } \\
\cline { 2 - 4 } & $\begin{array}{c}\text { Consumidores } \\
\text { violentos }\end{array}$ & $\begin{array}{c}\text { Consumidores No } \\
\text { violentos }\end{array}$ & $\begin{array}{c}\text { Consumidores } \\
\text { Abstinentes No } \\
\text { violentos }\end{array}$ \\
\hline Consumo de Cigarrillo durante el último año & 0.07 & 1.48 & -0.27 \\
\hline Consumo de Éxtasis durante el último año & 3.25 & 0.11 & 2.17 \\
\hline Consumo de Cacao Sabanero durante el último año & 2.35 & 0.11 & 1.15 \\
\hline Consumo de Heroína durante el último año & 4.45 & 0.11 & 2.17 \\
\hline Consumo de Hongo alguna vez en su vida & 1.58 & -0.12 & -0.56 \\
\hline Consumo de Marihuana alguna vez en su vida & 0.07 & -0.02 & -0.11 \\
\hline Consumo de Ácidos y Marihuana & 2.76 & 0.26 & -2.27 \\
\hline
\end{tabular}

Fuente: Programa estadístico R versión 3.3.2

Se presenta una caracterización de consumo de SPA por los grupos caracterizados anteriormente. Se evidenció que los consumidores de heroína, éxtasis, consumo simultáneo de ácidos y marihuana; y el cacao sabanero durante el último año, tienen una relación alta con el grupo de consumidores violentos, comparada con los otros dos, lo que revela sus comportamientos de violencia de manera efectiva, por el resultado estimulante, distorsionado que producen dichas sustancias en el organismo, así como los posibles efectos por el fenómeno de policonsumo (Tabla 6).

Resulta importante destacar que, sustancias de naturaleza estimulante en el sistema nervioso central, como la heroína y éxtasis, tienen la menor relación o influencia con el grupo de consumidores no violentos (0.11), comparado con los demás grupos de estudiantes, lo cual concibe un grupo menos excitado y con nula presentación de actos violentos. Del mismo modo, se asume la relación de los hongos y cacao sabanero que son de tipo alucinógenos, que hacen que este grupo de estudiantes no presenten actos de violencia de significancia.

Para el grupo de consumidores abstinentes no violentos, aunque las sustancias más relacionadas a su caracterización son la heroína, éxtasis y cacao sabanero durante el último año, su estimación no sigue siendo mayor. Es importante mencionar que, pese al consumo de estas SPA estimulantes, no muestran eventos de consumo simultáneo, lo que podría revelar que no existan comportamientos de violencia relevantes a nivel estadístico.

\section{Conclusiones}

Este estudio aporta a la descripción del panorama de consumo de sustancias psicoactivas y violencia en el contexto local y territorial.

Con relación a la prevalencia del consumo de SPA en los estudiantes, se encontró que, del total de participantes en el estudio, el $68.04 \%$ son consumidores al menos de una sustancia psicoactiva; valores que superan la mitad de la población abordada. Estos resultados no son ajenos a la realidad mundial y nacional, pues según la Organización Mundial de la Salud OMS (Observatorio de Drogas de Colombia, 2016), de 155 a 250 millones de personas entre los 15 y los 64 años habrían usado algún tipo de sustancia psicoactiva. En el análisis del Plan Nacional para la Promoción de la Salud y la Atención del Consumo de SPA en Colombia 2014-2021, se encontró un crecimiento del número de consumidores a nivel nacional, lo cual está relacionado a la diversidad de drogas disponibles (Ministerio de Salud y Protección Social, 2017). 
Las sustancias SPA mayormente consumidas son las bebidas alcohólicas y el cigarrillo, lo cual obedece a que son sustancias legales y su producción, venta y consumo no están prohibidos, aunque tienen ciertas restricciones en Colombia, según la Ley 124 de 1994(El Congreso de Colombia, 1994) y la Ley 1335 de 2009 (El Congreso de Colombia, 2009). De las SPA ilícitas, la marihuana se posiciona como la más consumida (14,63\%). Este hecho se agrava por el proceso de globalización, el cual facilita el marketing, diversificación y producción de este tipo de drogas e incrementa su demanda a lo largo de los diferentes grupos de población, incluyendo mujeres (Ministerio de Salud y Protección Social, 2016).

Llama la atención, que los rangos de edades de mayor consumo SPA es de 15 a 26 años (92.2\%) notando una participación entre menores de edad. Autores como García et al., (2017), confirman que edades comprendidas entre los 12 y los 29 años, experimentan una serie de cambios madurativos y psicológicos relacionados al trance de la etapa infantil a la adulta y en este proceso, se construye y perfila la identidad de los jóvenes y comportamientos que según sean, desencadenan procesos de salud- enfermedad. Es así como, los estilos de vida presentados respecto al consumo de SPA, los fines de consumo socio recreativo, la baja percepción en el nivel de riesgo y escasas alternativas de solución o ayuda frente al alto consumo, podrían ser factores que hacen más vulnerable a la población estudiantil universitaria a esta problemática. Respecto a esto, Amed et al. (2016), en el estudio efectuado en universitarios Sucreños sobre el consumo de SPA y sus estilos de vida, reportó que el $87 \%$ de los estudiantes, manifiesta tener una salud positiva y esta percepción de control los convierte en una población remisa a modificar las conductas de riesgo que practican, pudiendo desencadenar enfermedades a largo plazo (Amed et al., 2016).

Por otro lado, en el presente estudio se estima la relación del consumo de SPA y comportamientos violentos en los estudiantes de educación superior de Sincelejo ( $p=1.055 \mathrm{e}-7)$, lo que permite reconocer que el hecho de ser consumidor de sustancias psicoactivas puede generar comportamientos violentos. No obstante, al calcular la intensidad de la relación de dichas variables de estudio (Cramer's V=0,1977), se encuentra una relación positiva, pero débil, indicando que la presentación de los eventos violentos en esta población de estudio, podría obedecer a otros factores y no principalmente al consumo de sustancias psicoactivas.

En esto, la Organización Mundial de la Salud, establece tres factores que pueden incidir directamente con los actos de violencia en jóvenes: Factores de riesgo individual, como probemas de atención, hiperactividad y otros trastornos conductuales; delincuencia, consumo prematuro de drogas, bajo nivel intelectual y resultados académicos deficientes, fracaso escolar y desempleo. Dentro de los factores de riesgo en las relaciones cercanas, se encuentra escasa supervisión de los padres, prácticas disciplinarias severas, relajadas o incoherentes en el hogar, probemas afectivos de padres e hijos, abuso de sustancias o delincuencia de los progenitores e ingresos familiares bajos. Por último, en los factores de riesgo en la sociedad en general, se detalla el acceso consumo deliberado de alcohol, acceso a armas de fuego y su uso ilegal, pandillas y tráfico local de drogas ilícitas, gran desigualdad de ingresos económicos, pobreza, la baja calidad en el gobierno de un país, su legislación y aplicación así como la normativa en materia de educación y protección social (Organización Mundial de la Salud, 2020).

Por su parte, los comportamientos violentos que mostraron significancia estadística, aún cuando continúan bajo la modalidad de violencia verbal y física, su grado de severidad es mayor que los presentados desde la estadística descriptiva. Estos resultados coinciden con los hallados por Mesén, M. (2016), quien estudió la relación a partir del consumo de cada droga y 3 niveles de violencia (bajo, medio y alto), concluyendo que a mayor consumo de sustancias psicoactivas existe mayor violencia $(P=0,000)$. A su vez, ultimó que la presentación de los comportamientos violentos varía dependiendo de la droga que se está consumiendo. Esta apreciación guarda coherencia con los resultados del análisis de correspondencia múltiple de este estudio, en el que aparecen las sustancias de naturaleza estimulante, como la heroína y éxtasis como las más recurrentes en el colectivo 
universitario que presenta violencia, e incluso sin estar bajo efectos de la sustancia, lo que podría sugerir la presentación de cambios neuroquímicos y funcionales en el sistema nervioso central, por los efectos residuales que estas sustancias generarían a largo plazo.

Del mismo modo, Chavarriaga \& Segura en el 2015, determinaron que el consumo de SPA, se asocia con los comportamientos violentos del joven y representa un factor de riesgo para serlo, encontrando la heroína, la cocaína y éxtasis representan mayor riesgo que las demás SPA analizadas(Chavarriaga, M., Segura, 2015).

Se reportó además, la frecuencia de consumo de SPA como la variable que más distancia las personas violentas de las que no lo son. Se infiere que el efecto que según su naturaleza presenta cada sustancia SPA influye en la caracterización de grupos de estudiantes violentos o no. Asimismo, se induce que la relación entre consumo de SPA y violencia en los estudiantes universitarios del municipio de Sincelejo, podría estar influenciado por aspectos como: policonsumo simultáneo o consecutivo, edad de inicio del consumo, cantidad de sustancia SPA consumida, así como por factores culturales que no fueron valorados en este aparte.

Las edades con mayor compromiso a presentar comportamientos violentos tras el consumo de SPA, son desde los 23 a los 26 años con un $\mathrm{p}=1,57$ - -18 , lo que podría indicar que han venido presentando desde edades más tempranas el consumo y su efecto en el organismo cause abuso, dependencia y mayor irritabilidad frente a las demandas de su contexto social, académico, concurriendo en conductas de violencia.

Aunque hasta el momento, la literatura o las investigaciones estudiadas no ofrecen una delimitación y concordancia en los estratos socioeconómicos que más consumen SPA y presenten conductas de agresión, este estudio presentó con mayor relevancia al estrato 3, donde se asume que puede estar vinculado a las herramientas de medición del nivel de pobreza de los individuos, así como también a las particularidades de cada contexto socioeconómico y cultural, en concordancia con Cruz et al. (2017).

Los resultados encontrados en la presente investigación profundizan la necesidad de desarrollar acciones en materia de salud pública desde diferentes actores en este proceso: entidades gubernamentales, instituciones de eduación superior, autoridades y la familia como nucleo de la sociedad; todos alineados con el propósito de disminuir el consumo de SPA en los estudiantes, analizando los factores que más influyen en su ocurrencia, no sólo desde su producción y distribución, sino bajo una intervención interdisciplinar focalizada a las particularidades y determinantes intermedios de la salud en nuestro contexto, de tal modo que se consiga mayor sensibilización frente a este fenómeno sociosanitario.

\section{Referencias bibliográficas}

Amed, E., Mercado, J., González, M., Guerra, I., Viloria, J., y Támara, A. (2016). CONSUMO DE ALCOHOL, CIGARRILLO Y DROGAS COMO DETERMINANTE DE SALUD RELACIONADO CON LOS ESTILOS DE VIDA EN JÓVENES UNIVERSITARIOS.

Chavarriaga, M., Segura, A. (2015). Consumo de sustancias psicoactivas y comportamientos violentos en estudiantes de 11 a 18 años. Itagüí, Colombia. Revista de Salud Publica, 17(5), 655-666. https://doi.org/10.15446/rsap.v17n5.3136

Comisión Interamericana para el Control del Abuso de Drogas [CICAD]., Secretaría de Seguridad Multidimensional., y O. de los E. A. [OEA]. (2015). Comisión Interamericana para el Control del Abuso de Drogas-CICAD Secretaría de Seguridad Multidimensional Organización de los Estados Americanos-OEA.

Cruz, V., Gómez, C., y Rincón, C. (2017). SALUD MENTAL Y CONSUMO DE SUSTANCIAS PSICOACTIVAS EN ADOLESCENTES COLOMBIANOS. In Health and Addictions (Vol. 18, Issue 1). 
Díaz, V. (2009). METODOLOGÍA DE LA INVESTIGACIÓN CIENTÍFICA Y BIOESTADÍSTICA PARA PROFESIONALES Y ESTUDIANTES DE CIENCIAS DE LA SALUD. January.

El Congreso de Colombia. (1994). Ley_124_de_1994.

El Congreso de Colombia. (2009). ley1335 de 2009 cigarrillo.

García, V., Luque, B., Santos, M., Y., \& Tabernero, C. (2017). LA AUTORREGULACIÓN EMOCIONAL EN LA DEPRESIÓN Y EL DETERIORO COGNITIVO DE CONSUMIDORES DE SUSTANCIAS PSICOACTIVAS EMOTIONAL REGULATION ON DEPRESSION AND COGNITIVE DAMAGE OF PSYCHOACTIVE SUBSTANCE CONSUMERS. In Health and Addictions (Vol. 17, Issue 2).

Instituto Nacional de Psiquiatría Ramón de la Fuente Muñiz [INPRFM]. (2015). ENCUESTA NACIONAL DE CONSUMO DE DROGAS EN ESTUDIANTES.

Mesén, M. (2016). ASOCIACION ENTRE CONSUMO DE SUSTANCIAS PSICOACTIVAS Y ACTOS VIOLENTOS EN ESTUDIANTES DE 12 A 18 AÑOS DEL COLEGIO TÉCNICO PROFESIONAL MARIO QUIRÓS SASSO EN EL MES DE MAYO DEL EL AÑO LECTIVO 2016.

Ministerio de Defensa. (2017). Indicadores de seguridad. Dirección de Estudios Estratégicos Grupo de Información Estadística.

https://www.mindefensa.gov.co/irj/go/km/docs/Mindefensa/Documentos/descargas/estudios_sectoriale s/deptos/NARINO.pdf

Ministerio de Salud y Protección Social. (2016). ESTUDIO DE EVALUACIÓN Y DIAGNÓSTICO SITUACIONAL DE LOS SERVICIOS DE TRATAMIENTO.

Ministerio de Salud y Protección Social. (2017). PLAN NACIONAL PARA LA PROMOCIÓN DE LA SALUD, LA PREVENCIÓN, Y LA ATENCIÓN DEL CONSUMO DE SUSTANCIAS PSICOACTIVAS 2014-2021.

Observatorio de Drogas de Colombia. (2016). Reporte de Drogas 2016. In Revista Brasileira de Ergonomia (Vol. 9, Issue 2). https://doi.org/10.5151/cidi2017-060

ODC. (2013). Estudio Nacional De Consumo De Sustancias Psicoactivas En Colombia - 2013. Observatorio de Drogas de Colombia, 66, 37-39. www.odc.gov.co

Organización Mundial de la Salud. (2020). Violencia juvenil.

Ortega, Y., Hernández, A., Arévalo, A., Díaz, A., y Bermúdez, Z. (2018). Causas y consecuencias del consumo de sustancias psicoactivas en adolescentes con ambientes de vulnerabilidad familiar y contextos sociales conflictivos. Concejo de Bogotá, 70. http://concejodebogota.gov.co/causas-y-consecuencias-del-consumode-sustancias-psicoactivas-en-jovenes/cbogota/2018-07-04/155943.php

Pérez, A., Scoppetta, O. (2008). Consumo de alcohol en menores de 18 años en Colombia 2008: un estudio con jóvenes escolarizados de 12 a 17 años en 7 capitales de departamento y dos municipios pequeños.

UNODC. (2017). III ESTUDIO EPIDEMIOLÓGICO ANDINO SOBRE CONSUMO DE DROGAS EN LA POBLACIÓN UNIVERSITARIA DE COLOMBIA, 2016.

UNODC, (United Nations Office on Drugs and Crime). (2016). Informe mundal sobre las drogas 2016. Journal of Chemical Information and Modeling, 53(9), 16. https://doi.org/10.1017/CBO9781107415324.004

UNODC, (United Nations Office on Drugs and Crime). (2018). World Drug Report 2018 RESUMEN

(CONCLUSIONES). World Drug Report 2018, 1-10.

https://www.unodc.org/wdr2018/prelaunch/WDR18_ExSum_Spanish.pdf

Esta obra está bajo una Licencia Creative Commons Attribución-NoCommercial 4.0 International

$(\mathrm{Cc}) \mathrm{BY}$-NO 